\title{
Chagas disease by oral transmission: cardiovascular manifestations
}

\begin{abstract}
Introduction: Chagas disease is the most common cause of myocarditis in South, Central and parts of North America. More than 10million Latin Americans carry the protozoan parasite Trypanosoma cruzi, 25 millions of people at risk to acquire the disease and at least one million of them will die. Transmission of parasitosis habitually depends on the participation of infected triatomines. However, acute disease caused by the accidental ingestion of contamined food, either by feces of triatomines or anal secretion of marsupials has received considerable attention recently. A sparse number of outbreaks of orally acquired human Chagas disease have been reported from Brazil, Argentina, Colombia and Venezuela. The findings in multiple outbreaks established the absence of Romaña's sign or any external point of $T$. cruzi entrance, presence of simultaneous onset of Chagas disease acute symptoms in members of the same family or community. The clinical manifestations are prolonged fever, dyspnea, myalgias, headache, facial and limb edema. The cardiovascular findings are myocarditis, acute congestive failure, arrhythmias, and sudden death with high mortality rate estimated at $8-35 \%$. Diagnosis can be made by direct methods, serologic techniques, inoculation in mice of culture blood, and polymerase chain reaction. In the follow up of these patients to prevent Chagas cardiomyopathy, it is important to perform echocardiographic and electrocardiographic evaluation to keep a close eye on systolic and diastolic biventricular function and abnormalities in 12-lead electrocardiogram in asymptomatic patients. Treatment of this disease use nitro derivates compounds, to avoid the parasite proliferation but aren't able to eliminate the entire population of T.cruzi.
\end{abstract}

Conclusion: Changes in the strategy of Chagas disease control programs are mandatory; which until now are limited to vector control activities in rural Latin American communities in endemic areas.

Keywords: chagas disease, oral transmission, triatomines, myocarditis, chagas cardiomyopathy
Volume 8 Issue I - 2017

\author{
Rojas Cielo,' Machado Ivan,' Alarcon de \\ Noya Belkisyole ${ }^{2}$ \\ 'Department of Cardiology, Congenital Heart Disease Section, \\ Hospital Universitario de Caracas, Universidad Central de \\ Venezuela,Venezuela \\ ${ }^{2}$ Department Parasitology, Inmunology Section, Tropical \\ Medicine Institute, Universidad Central de Venezuela, Venezuela
}

Correspondence: Cielo Rojas, Department of Cardiology, Congenital Heart Disease Section, Hospital Universitario de Caracas, Universidad Central de Venezuela,Venezuela, Tel 0058 4168348343, Fax 0058-2 I27256, Email cielitorojas@gmail.com

Received: December 20, 2016 | Published: January 20, 2017
Abbreviations: $\mathrm{ChD}$, chagas disease; $T$. cruzi, trypanosoma cruzi; IgG, inmunoglobulin G; IgM, Inmunoglobulin M; ELISA, enzyme-linked immune absorbent assay; IH, indirect hemaglutination; $\mathrm{WB}$, western blot; PCR, polymerase chain reaction; BNP, brain natriuretic peptide; RV, Right ventricle; ECG, electrocardiogram

\section{Introduction}

Chagas disease $(\mathrm{ChD})$ or American trypanosomiasis since its discovery by Carlos Chagas nearly 100years ago is the most common cause of myocarditis in South, Central and parts of North America. More than 10million Latin Americans carry the protozoan parasite Trypanosoma cruzi, 25millions of people are at risk to acquire the disease and at least one million of them will die, within a 10year mortality of $84 \%$, unless new strategies for diagnosis, treatment and increased access to medical care are taken. ${ }^{1}$ With the migration of infected individuals from Latin America to the United States, the Center for Disease Control and Prevention (CDC) estimates that more than 300.000 persons with T.cruzi infection live in this country, where it now poses a major public health concern because of its rising prevalence and limited awareness within the medical community. Through both globalization and immigration the prevalence of $\mathrm{ChD}$ in non endemic countries is expected to increase. ${ }^{2}$

\section{Epidemiology}

This pathology affects impoverished people in the Americas from Mexico to north of Argentina. The main vectors are triatomine insects, particularly Triatoma infestans, Rhodnius prolixus and Patrongylus geniculatus, popularly known as the kissing bugs. ${ }^{3}$ They live in thatches roofs and cracks in the walls of poorly constructed homes. Success control of $\mathrm{ChD}$ was achieved by regional programs, like campaigns based on the use of residual pesticides and shifted mud houses for brick houses (favelas, shacks, slums) to reduce triatomine vectors. These programs reduced the transmission from 700.000 annually new cases in 1990 to 42.200 in 2006 . Since rural to urban migrations have occurred in the $60 \mathrm{~s} \mathrm{ChD}$ began to be diagnosed in the cities and periurban regions. These demographic changes have reversed the proportion of the urban vs. rural population, from $70.5 \%$ and $47 \%$ in 1950 to $15 \%$ and $11.2 \%$ in Brazil and Venezuela, respectively. The exodus of Latin Americans to more developed countries, accounts for the estimates 100.000 or more chronically infected persons now living in the United States and Europe. ${ }^{2}$ Despite sanitary measures that have reduced transmission rates through vector control and serological screening of blood donors, Chagas disease persist as a public health problem, observing a progressive increase in cases in the Amazon basin. ${ }^{4}$

\section{Pathogenesis}

Transmission of parasitosis habitually depends on the participation of infected triatomines, $T$. cruzi is excreted in the feces of infected triatomine onto human skin or near mucous membranes. The parasites breach the dermis trough excoriations in the skin and gain systemic access. Once within host cells they reproduce, which leads to cell lysis and hematogenous dissemination. When triatomine vector ingesting $T$. 
cruzi from the blood of human host, the cycle is complete. ${ }^{2}$ Chagas disease can also be transmitted by alternative mechanisms such as blood transfusion, organ transplants, and vertical transmission or by accidental contamination in a laboratory in persons handling tissue or the blood from infected animals. A mechanism, presumed to be frequent among wild animals, is the ingestion of triatomines; therefore it is possible that the human can acquire the infection when accidentally consuming beverages or contaminated food with vectors or their feces. ${ }^{4}$ The latter route has received considerable attention recently. ${ }^{5}$

Cardoso et al., ${ }^{3}$ evaluate the survival of Trypanosoma cruzi contaminating sugar cane used to prepare juice, and the viability and capacity for infection by the parasite after recovery. In this essay they demonstrated the high survival rate of T. cruzi in sugar cane by direct analysis (up to 12hours), hematocrit tube centrifugation test (up to 4hours) and acridine orange Quantitative Buffy Coat, (up to 12hours) and experimental inoculation in BALB/c mice (showed parasitemia during 14day observation period). ${ }^{3}$

In the classic way of transmission, inoculation is followed by an incubation period of 1 to 2 weeks after which the infection can be expressed with unspecific symptoms or asymptomatic patients in the acute presentation. In the acute phase of Chagas disease, cardiac involvement can occur in up to $90 \%$ of cases and after 6 to 9 weeks most patients show recovery of the clinical picture. ${ }^{2}$ Following the acute phase, about $30 \%$ of patients directly enter the symptomatic chronic phase, but the majority of patients proceed to the indeterminate phase, when the infestation is only diagnosed by serological or parasitological tests; subclinical cardiac involvement may be demonstrated by imaging studies in some patients. ${ }^{6}$ The latter phase involves host-parasite equilibrium and is without progressive host damage. Approximately one-third of patients progress from indeterminate to chronic phase in which the heart and gastrointestinal tract are the principal targets, and symptoms do not appear for $15-30$ years. ${ }^{7}$ For poorly understood reasons, $10-30 \%$ of infected patients have clinical manifestations in chronic Chagas disease, 30\% develop cardiac abnormalities and more than $10 \%$ digestive and neurological problems. Inflammation and destruction of autonomic ganglia lead to megaesophagus dysphagia and prolonged megacolon constipation. Heart disease is the more common and severe manifestation; cardiac symptoms and signs arise from progressive myofibril fibrosis and conduction system injury. Chronic inflammatory infiltrates, diffuse and local fibrosis, and loss of myocardial cells caused by direct aggression by the parasite, sympathetic and parasympathetic dysautonomia and coronary microvascular abnormalities due to an autoimmune processes, lead to dilated chronic Chagasic cardiomyopathy. ${ }^{6}$

Anez et al., ${ }^{8}$ reported in 2016 the presence of Trypanosoma cruzi in creviscular fluid from gingival inflammation foci of chronic chagasic patients. Samples taken from 11 unquestionable chronic chagasic patients who had suffered from acute infection between 7 months and 27years before the endpoint evaluation, revealed the presence of specific T. cruzi - DNA in all of them. This fact demonstrates that T. cruzi persistence in human hosts is a long-standing phenomenon and the oral tissue is frequently involved. This phenomenon occurred independently of the degree of gum inflammation, the time of infection and/or the patient clinical profiles. ${ }^{8}$

\section{Clinical manifestations}

Acute disease caused by the accidental ingestion of contaminated food, either by feces of triatomines or anal secretion of other vectors such as marsupials, has an incubation period of approximately 5-20days. The clinical manifestations are prolonged fever (more than 7days), ${ }^{9}$ dyspnea, myalgias, headache, facial and limb edema and the absence of parasite entrance door sign (inoculation Chagoma inflammatory nodule at site of inoculation or Romaña sign - periorbital swelling). In $<5 \%$ of cases, more severe illness, myocarditis, and acute congestive heart failure with high mortality estimated at $8-35 \%$, can occur. ${ }^{10}$ In myocarditis, the electrocardiogram may show sinusal tachycardia with nonspecific ST-segment and T-wave abnormalities, occasionally changes are suggestive of an acute myocardial infarction and pericarditis not infrequently accompanies this entity. In most cases, when there are nonspecific clinical manifestations, not always associated with the cardiovascular system, epidemiological suspicion is a challenge for the clinician; especially in the urban environment where Chagas disease has not been routinely considered as differential diagnosis (Table 1). ${ }^{11}$

After the acute phase of the disease, most patients enter in a clinically asymptomatic chronic phase (indeterminate phase). This phase generally carries an excellent prognosis with survivals rates similar to those of noninfected individuals. For infected individuals, $20-30 \%$ will progress from the indeterminate to the symptomatic chronic phase. In this one, progression of cardiomyopathy leads to cardiac dilation and biventricular failure. ${ }^{12}$ Other findings of advanced heart disease include left ventricular apical aneurysm, complex ventricular arrhythmias, sinus bradyarrhytmias, and intraventricular and atrioventricular conduction block. Right bundle-branch block, left anterior hemiblock and prolongation of the QRS complex precedes other manifestations of chronic cardiomyopathy. The most common cardiovascular cause of death is sudden death, progressive heart failure, and embolism to multiple organs. ${ }^{9}$ Sudden death accounts for $\square 60 \%$ of the mortality due to chronic $\mathrm{ChD}$, whereas heart failure causes $25-30 \%$ of deaths, and stroke is responsible for $10 \%$ to $15 \%$. The electrocardiogram provides prognostic information, infected patients $<60$ years old with normal ECG have long-term survival rates similar to seronegative Chagas patients. ${ }^{13}$

Table I Diagnostic key points for oral transmitted Chagas disease ${ }^{14}$

\section{Simultaneity of similar symptoms among several persons}

Affected individuals who have shared food or beverage at the at the same time Exposure to handmade beverages in endemic areas

Absence of clinical signs of parasite entrance (Romaña or Chagoma signs) Laboratory screening for Chagas disease in long lasting high fever, facial or lower limb edema, decay, myocarditis and other unspecific symptoms

Demonstration of motile Trypanosoma cruzi blood trypomastigotes in an acute outbreak in spite of being asymptomatic patients

Serological tests (specific IgM and IgG detection by ELISA) for the rapid screening of large groups of suspected infected individuals

Retrospective demonstration of concomitant symptoms in related individuals from a recent index chronic case of Chagas disease

\section{Diagnosis}

The diagnosis of $\mathrm{ChD}$ for oral transmission can be made by direct methods like fresh and Giemsa-stained peripheral blood smears for review for trypomastigotes, inoculation of blood patient that were cultures in biphasic medium and inoculated intraperotoneally in mice, screen for immunoglobulin $\mathrm{G}$ ( $\operatorname{IgG}$ ) and immunoglobulin $\mathrm{M}(\operatorname{IgM})$ antibodies against $T$. cruzi by serological techniques like enzymelinked immunoabsorbent assay (ELISA), indirect hemaglutination $(\mathrm{IH})$, and Western blot (WB) test, and evaluation by a polymerase chain reaction (PCR). ${ }^{14}$

Considering Chagas disease in the context of inflammatory diseases, biomarkers of heart muscle injury like troponin I has been 
evaluated. In chagasic individuals, the concentration of troponin I has been indentified; being a prognostic marker of myocardial damage degree and consequently risk of death. The brain natriuretic peptide (BNP), a neurohormone of specific cardiac origin has been associated with cardiac dysfunction with discordant results. A cutoff lower than $60 \mathrm{pg} / \mathrm{ml}$ of serum BNP characterizes patients with low risk of severe cardiomyopathy. The assessment of systolic and diastolic functions, right atrial pressure with serum BNP levels helps to stratify the risk to develop of cardiovascular events. ${ }^{9}$

Echocardiography is a useful tool to evaluate myocarditis in the acute phase of ChD. Segmental or global wall motion abnormalities in myocarditis can simulate myocardial infarction. Pericardial effusion can be observed in $40 \%$ to $50 \%$ of these cases. Increased sphericity and left ventricular volume can occur and loss of right ventricular function is a predictor of death in these patients. ${ }^{10}$ Chronic Chagas cardiomyopathy constitutes a serious public health problem as it is a major cause of heart failure with a high mortality rate in chronic phase. The identification of this pathology conferred poor prognosis for patients with heart failure, regardless of clinical and echocardiographic data. The study of right ventricular function in the follow up of patients with Chagas for oral transmission may be useful for risk stratification of Chagas cardiomyopathy. The myocardial performance index (tei index), allows analysis of systolic and diastolic function of the right ventricle (RV) and adds significant prognostic information. Another index used to evaluate RV systolic function is the tricuspid annular plane systolic excursion (TAPSE), providing more reliable information. ${ }^{7}$ Changes in the RV morphological and functional characteristics are associated with significant left ventricular involvement and pulmonary hypertension, contributing to poor prognosis and acting as an independent determinant of reduced exercise capacity. ${ }^{15}$

Left ventricular wall motion abnormalities in patients with normal systolic function were shown to be a predictor of ventricular function deterioration in chagasic patients with normal ECG and no symptoms or physical signs. The presence of apical aneurysm, impaired left ventricular function, increase left atrial volume and ventricular extrasystoles also are predictors of poor prognosis. Mitral annular tissue Doppler asses ventricular diastolic and systolic function, disclosed early abnormalities of myocardial contractility. The E/E ratio showed high prognostic value for mortality in patients with mild or moderate left ventricular dysfunction. Finding dyssynchrony, being a segmental alteration secondary to the presence of areas of fibrosis associated with chronic diffuse inflammatory process, does not behave as a risk factor. ${ }^{9}$

The two dimensional X-strain echocardiography is a technique that allows differentiating passive from active deformation of the ventricular cavity walls, though the assessment of tangential and longitudinal deformation. This technique showed the presence of significant reduction in the percentage of deformation and the rate of deformation of the infer-lateral left ventricular wall, especially in the middle and apical portions, regions that show segmental contractility alterations in the cardiac form of Chagas disease. The use of the speckle tracking technique, allows determination of basal and apical myocardial rotation and the twisting (the angular difference resulting from the clockwise rotation of the apical region and of the basal in the counterclockwise direction during ventricular systole). In the chagasic patient we can identify the reduction in global longitudinal strain, radial strain, twisting and myocardial rotation. ${ }^{15}$

Cardiac autonomic function can be evaluated using angiography with Tc-99 labeled RBCs, demonstrating parasympathetic cardiac injury and right ventricular dysfunction in the absence of diagnosed left ventricular dysfunction. The association of autonomic dysfunction with normality of most ventricular echocardiographic variables suggests that dysautonomia must be a primary phenomenon that may precede mechanical changes; constitute a precursor of ventricular dysfunction. ${ }^{9}$

\section{Treatment}

The use of specific drugs (Nitro derivates compounds) associated with the host's immune anti-T. cruzi response may keep the disease in check, avoiding parasitic proliferation, none of them are able to eliminate the entire population, allowing tissue parasite to persist for long periods of time during the chronic phase. ${ }^{4}$ The drugs available are benznidazole at a dosage of $6 \mathrm{mg} / \mathrm{kg} /$ day for 60days or nifurtimox at a dosage of $8 \mathrm{mg} / \mathrm{kg} /$ day for 90 days. ${ }^{16}$ Both benznidazole and nifurtimox have significant side effects, but early discontinuation of the latter has been more frequent. Benznidazole side effects are skin rash, photosensitivity (50\% cases), polyneuritis (10-30\%), headache, anorexy, abdominal pain, chest pain, palpitations, dizziness, nausea, granulocytopenia, leucopenia, thrombocytopenia, insomnia, disorientation and seizures. Nifurtimox side effects are nausea, vomiting, diarrhea, dermatitis, fever, peripheral neuropathy, tachycardia, neuropsychiatric disorders, jaundice, headache, leucopenia, thrombocytopenia and hemolysis. Although neither drug is approved for use in the United States, both can be obtained from the $\mathrm{CDC}$ and used under investigational protocol. ${ }^{17}$

\section{Discussion}

A sparse number of orally acquired human $\mathrm{ChD}$ outbreaks have been reported from Brazil, Argentina and Colombia (Brazil 1965 Teotonia Río Grande do Sul 17 cases, Brazil 2005 Navegantes Santa Catarina 31 cases, Colombia 1999 Magdalena 18 cases). ${ }^{18}$ Venezuela has a successful $\mathrm{ChD}$ vector control program based on the improvement of rural housing and vector control. However, epidemiological data suggest a reemergence of the infection. A preponderance of cases occurring among adults with relatively high lethality (up to $35,2 \%$ ) and occurrence in remote rural areas or in urban communities where fruits obtained from areas of endemicity, such as açai (Euterpe oleracea), piassava (Leopoldinea piaçaba), and sugar cane, were consumed. The persistence of numerous sylvatic foci and the wide distribution of vectors and reservoirs, with a progressive reduction in the availability of the vector's natural source of blood (birds and mammals) in intervened forested areas, are driving originally wild triatomines to invade human dwellings. Once domiciliation has occurred, $P$. geniculatus may feed abundantly on domestic reservoirs, as well as on humans (Figure 1). Vectors eventually contaminate unprotected food and beverages with feces and there is also the possibility of transmission by food contamination with urine or anal secretions of infected marsupials. ${ }^{19}$

In Caracas, a traditionally non-endemic area, three children have been reported in the last ten years due to acute manifestations of Chagas disease; acquired the first two by vector transmission in 1999 an 2005 respectively, and the last one in the largest urban outbreak by oral transmission occurred in a middle class school in $2007 .^{20}$ In this urban outbreak 103 people were infected by the ingestion of guava juice prepared in inadequate sanitary conditions, established during the epidemiological study, in which 22 were hospitalized and one child died with acute myocarditis. Fever $(81 \%)$ and facial edema $(40,9 \%)$ were the most common initial signs. Other common findings were headache (36\%), asthenia (36\%) and myalgia (31\%). Symptoms and signs of cardiovascular disease were reported, including 
precordial chest pain, pericardial effusion, cardiomegaly and a case of atrial fibrillation. ${ }^{17}$ In $61(59 \%) \geq 1$ abnormalities was noticed on the ECG recordings, T wave abnormalities were significantly more common among patients $\leq 18$ years old, whereas supraventricular arrhythmias and microvoltages were predominant among adults, who more frequently develops severe clinical cardiological manifestations that required hospitalization. ${ }^{13}$ The differential diagnoses of these patients were dengue, hystoplasmosis, mononucleosis, polymyositis, autoimmune disease and fever of unknown origin. Laboratory findings were leukocytosis, increase of acute phase reactants (globular sedimentation velocity and reactive C protein), troponin I and LDH.

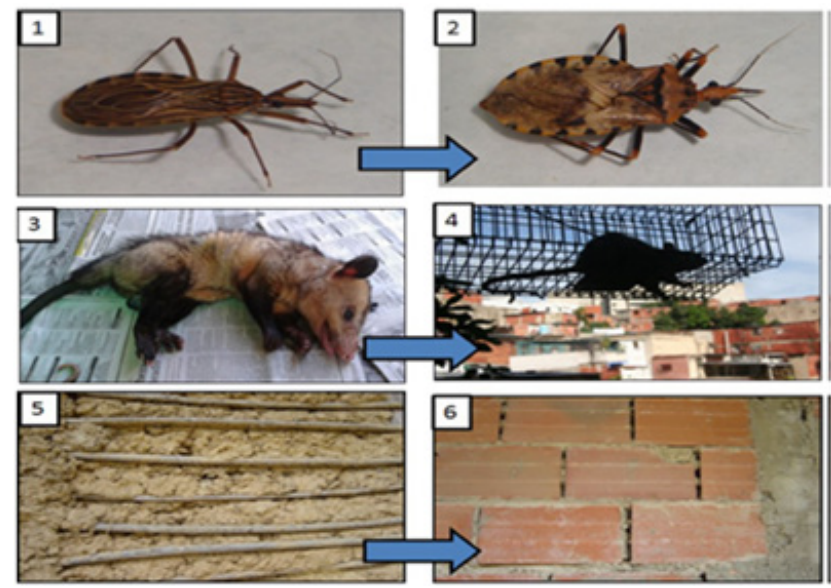

Figure I Ecological, biological and demographic factors that favor oral transmission of Trypanosoma cruzi to humans.

I. Rhodnius prolixus, important vector responsible for cutaneous transmission. 2. Pastrongilus geniculatus primarily vector incriminated in oral transmission. 3. Didelphis marsupialis, important wildlife reservoir.

4. Rattus rattus, urban reservoir 5.Wall mud dwelling in rural areas 6. No fleecy wall where domiciled triatomine can live in urban areas. ${ }^{22}$

Fifty-one of the 103 infected patients were followed up 5 years after this outbreak, with a second cycle of treatment with nifurtimox or benznidazole in $43 \%$ of them. The resolution of the echocardiographic findings was observed in $100 \%$ of the patients with persistent electrocardiographic alterations; abnormalities in $\mathrm{T}$ wave (35\%), ST segment elevation (4\%), non specific intraventricular conduction disorders $(22 \%)$ and sinus bradycardia $(4 \%){ }^{20}$ The echocardiographic and electrocardiographic findings of this group of patients are currently being studied in 9years of follow-up (in process of publication). Another micro-epidemic outbreak associated with Trypanosoma cruzi oral transmission, detected in a rural locality of Merida, Venezuela was published in 2016. The presence of similar clinical profiles, massive active parasitemia, specific anti-T.cruzi IgM and infection by the same genetic T. cruzi genotype detected in 9 patients, with absence of Romaña's sign, indicate simultaneous infections by ingesting T.cruzi contaminated food. The clinical findings in these patients were fever not responsive to antibiotic or antipyretic treatment, headache and myalgia. In the four fatal cases (44\%) hepatomegaly was present $(100 \%)$ and myocarditis, pericardial effusion and cardiomegaly were respectively detected in $75 \%, 50 \%$ and $25 \%$ of them. Histopathological observations revealed the presence of large number of T. cruzi-tissue forms and massive lymphomonocytic infiltration in samples from heart, liver, spleen, skeletal muscle and tongue of fatal case. ${ }^{21}$

In these patients, the early etiological diagnosis of chagasic cardiomyopathy enables implementation of measures to reduce mortality offering new possibilities for a prolonged quality of life. Progressive environmental changes have favored the urbanization and domiciliation of the cycle maintained by $P$. geniculatus, thus affecting the poor populations of the misery belts around most Latin-American cities and middle-class populations. ${ }^{22}$ This situation imposes necessary changes in the strategy of $\mathrm{ChD}$ control programs, which until now have been limited to vector control activities in rural Latin American communities in endemic areas. ${ }^{23}$

\section{Conclusion}

The findings in multiple reported outbreaks established the absence of Romaña's sign or any external point of T. cruzi entrance in the patients; presence of simultaneous onset of Chagas disease acute symptoms in members of the same family with similar frequency, history and severity, massive detection of blood circulating parasites and similar T. cruzi genotype found in all the examined samples, detection of specific anti-T. cruzi IgM in all patients and high lethality. All these findings led to reach the conclusion of the occurrence of an orally transmitted acute Chagas disease. Chagas disease by oral transmission should be included in the routine laboratory study of prolonged febrile syndrome. It is vital to add this disease to the epidemiological surveillance in Latin American countries; including an adequate handling of prevention protocol of food and beverages. And also to include Chagas disease among food transmission diseases. Changes are necessary in the strategy of $\mathrm{ChD}$ control programs, which until now are limited to vector control activities in rural Latin American communities in endemic areas.

\section{Acknowledgments}

None.

\section{Conflicts of interest}

Author declares there are no conflicts of interest.

\section{Funding}

None.

\section{References}

1. Maguire J. Chagas' Disease - Can we stop the deaths? $N$ Engl $J$ Med. 2006;355(8):760-761.

2. Malik L, Singh G, Amsterdam E. The Epidemiology, Clinical Manifestations, and Management of Chagas Heart Disease. Clin Cardiol. 2015;38 (9):565-569.

3. Cardoso A, Lescano S, Amato V, et al. Survival of Trypanosoma cruzi in sugar cane use to prepare juice. Rev Inst Med Trop S Paulo. 2006;48(5):287-289.

4. Alarcón de Noya B, Veas J, Ruiz-Guevara R, et al. Evaluación clínica y de laboratorio de pacientes hospitalizados durante el primer brote urbano de enfermedad de Chagas de transmisión oral en Venezuela. Rev Patol Trop . 2013;42(2):177-186.

5. Martín A, Alarcón de Noya B, Montero R, et al. Epidemia de Chagas agudo adquirido por vía oral en una escuela de Caracas: Descripción del caso índice. Arch Venez Puer Ped . 2009;72(3):97-100.

6. Alarcón de Noya B, Torres J, Suárez J, et al. Guía para el diagnóstico, manejo y tratamiento de Chagas en fase aguda a nivel de los establecimientos de salud. Avances Cardiol. 2008;28(4):250-267.

7. Marín Neto J, Rassi A. Pathogenesis and prognostic factors in chronic Chagas cardiomyopathy. Rev Esp Salud Pública. 2015;11-21. 
8. Anez N, Crisante G, Khoudeir S, et al. Trypanosoma cruzi Persistence in creviscular fluid from inflamed gum of chronic Chagasic patients. $J$ Dent Oro Surg . 2016;1(5):123-127.

9. Clodoval de Barros PJ, Brivaldo MF. Clinical and Echocardiographic pedictors of mortality in Chagasic cardiomyopathy-Systematic Review. Arq Bras Cardiol . 2014;102(6):602-610.

10. Cooper L. Myocarditis. N Engl J Med. 2009;360(15):1526-1538.

11. Parada H, Carrasco H, Añez N, et al. La enfermedad de Chagas aguda. Características clínicas, parasitológicas e histopatológicas. Avances Cardiol. 1996;16(1):10-17.

12. Noya O, Ruiz-Guevara R, Díaz Bello Z, et al. Epidemiología y Clínica de la transmisión oral de Trypanosoma cruzi. Rev Esp Salud Pública. 2015;23-34.

13. Alarcón de Noya B, Díaz-Bello Z, Colmenares C, et al. Large outbreak of orally acquired acute Chagas Disease at School in Caracas, Venezuela. JID. 2010;201(9):1308-1315.

14. Alarcón de Noya B, Díaz-Bello Z, Colmenares C, et al. Update on oral Chagas disease outbreaks in Venezuela: epidemiological, clinical and diagnostic approaches. Mem Inst Oswaldo Cruz. 2015;110(3):377-386.

15. Acquatella H, Puigbó J, Gómez-Mancebo J, et al. Utilidad de la ecocardiografía/Doppler en la enfermedad de Chagas. Gac Méd Caracas. 2011;119(4):274-286.

16. García R, Hernández E, Rodríguez-Bonfante C, et al. Primer Consenso venezolano sobre la enfermedad de Chagas: Conclusiones y recomendaciones. Avances Cardiol . 2001;21(1):14-23.
17. Miles M. Orally Acquired Chagas Disease: Lessons from an Urban School Outbreak. JID. 2010;201(9):1282-1284.

18. Pinto J. O Trypanosoma cruzi e suas características bio-ecológicas, como agente de enfermedades transmitidas por alimentos. Informe de la consulta técnica en epidemiología, prevención y manejo de la transmisión de la enfermedad de Chagas como enfermedad transmitida por alimentos (ETA). OPS/OMS. 2006; 27-30.

19. Rojas C, Martín A, Machado I, et al. Acute Chagas disease by oral transmission in children. Cases reported in urban population: Caracas, Venezuela. 5th World Congress of Paediatric Cardiology and Cardiac Surgery, Australia. 2009.

20. Bolívar M, Elías M, Marques J, et al. Five years of cardiologic followup of children with acute Chagas diease infected by the oral route in Venezuela. 47th Annual meeting of the association for European Pediatric and Congenital Cardiology (AEPC). London. 2013.

21. Anez N, Crisante G, Rojas A, et al. A new acute oral Chagas disease outbreak in Merida, Venezuela: A comprehensive study. International Journal of Clinical Medicine Research. 2016; 3(1):29-37.

22. Alarcón de Noya B, Noya O. An ecological overview on the factors that drives to Trypanosoma cruzi oral transmission. Acta Tropica. 2015;151:94-102.

23. Alarcón de Noya B, Díaz-Bello Z, Colmenares C, et al. Enfermedad de Chagas de transmisión oral: vinculación del caso índice con una microepidemia urbana en Venezuela. Bol. Mal. Salud Amb. 2010;15(1):135-138. 